\title{
Trisomy 18 Syndrome: An Ethical Dilemma in the Neonatal Intensive Care Unit
}

\section{Andrea Caines}

Department of Nursing, Barry University, USA

*Corresponding author: Andrea Caines, Department of Nursing, Barry University, USA, Tel: 9543296502; E-mail: anncaines@aol.com

Received date: July 31, 2018; Accepted date: November 14, 2018; Published date: November 19, 2018

Copyright: $\odot 2018$ Caines A. This is an open-access article distributed under the terms of the Creative Commons Attribution License, which permits unrestricted use, distribution, and reproduction in any medium, provided the original author and source are credited.

\section{Introduction}

Healthcare professionals, patients and their families face difficult decisions regarding medical treatments, which may involve moral principles, professional standards, and personal religious beliefs. Ethical issues in healthcare arise daily when physicians, nurses and other member of the healthcare team have to make tough decisions that will affect a patient's life and/or health and wellbeing. These decisions most often involve what is in the best interest for the critically ill individual when the odds are unclear. It is that power over an individual's life that has led healthcare organizations to implement an ethical board or committee that has an established code of ethics that acts as a guide in the ethical decision-making process. Within the Neonatal Intensive Care Unit (NICU) ethical dilemmas are prevalent and usually result when there is a disagreement between the physician and the parents over what is in the best interest, and who should have the authority to make that decision. The purpose of this position paper is to investigate an ethical dilemma in the NICU, present two opposing perspectives on the issue, and then the author of this paper will identify and justify their position on the issue.

Congenital malformation such as trisomy 18 and 13, are frequently referred to as 'incompatible with life' or at 'lethal malformation'. Trisomy 18, also known as Edwards syndrome, is a congenital disorder that occurs because of the presence of an extra 18 chromosome; individuals with this condition have three copies of chromosome 18, instead of the usual two copies [1]. Trisomy 18 is the second most common congenital trisomy condition after Down's syndrome (Trisomy 21); accounting for approximately one in 4,000-8,500 live births [2]. Infants with trisomy 18 disorder present with distinctive physical features such as low-set ears, rocker bottom feet, and a short neck [2]. Other anomalies include cardiac ventricular septal defect, pulmonary hypertension, brain malformations such as microcephaly, central apnea, feeding difficulties related to esophageal atresia, and malrotation of the intestines [2]. However cardiac failure as a result of cardiac malformations is the major cause of death in children with Trisomy 18 [3]. $80 \%$ to $100 \%$ of children with Trisomy 18 syndrome Due to these conditions medical professionals have often taken a stance as to not aggressively treat these infants who survive labor and delivery and the immediate newborn person [2]. Today, in NICU's across the United States physicians and healthcare professionals caring for infants with trisomy 18 have accentuated comfort care over more aggressive forms of treatment such as a cardiac surgical repair Research studies have shown that if an infant with Trisomy 18 survives beyond the first weeks after birth, then the chances of surviving beyond their first year increases to approximately $5 \%$ to $10 \%$ [1]. One of the most challenging ethical dilemmas in neonatal medicine is whether to offer aggressive resuscitative and surgical treatment for infants diagnosed as having a poor prognosis because of genetic anomalies, such as trisomy 18 . Physicians are faced with knowing that this congenital anomaly is not compatible with life while families of the child want medical and surgical intervention. The argument ensues whether to provide aggressive medical interventions or provide comfort care. We will address both positions, whether to medically intervene or due to the low survival rate along with the severe disabilities associated with Trisomy 18, withhold medical intervention. We will start by looking at the perspective of the physicians and healthcare professionals.

\section{No Medical Intervention}

In cases of trisomy 18, decisions of whether to limit medical interventions rely on an assessment of many factors that must be considered from both the healthcare providers' and families' perspectives. When these ethical challenges arise decisions and questions to address are the patient's quality of life, patient sufferings, the allocation of resources are all things that require great consideration when making these life or death decisions. Medical science has identified that the chromosomal anomaly in Trisomy 18 has labeled the condition not compatible with life.

Along with the complex complications associated with this disorder, contribute to a short life expectancy in patient with Trisomy 18. As a result of the lethal nature of Trisomy 18, physicians and healthcare professionals have decided not to aggressively intervene instead provide comfort care. Aside from the incompatibility of life diagnosis, Healthcare providers consider other factors before deciding whether or not to treat the patient with Trisomy 18: Chances that the intervention (i.e., surgical) will succeed, weighing the risk involved with intervention versus non-intervention, the pain and long term effects/ complication associated with an intervention, and finally what would be the quality of life for the infant if an intervention is implemented or withheld. According to the literature many children born with Trisomy 18 die within the first year of life [1]. Therefore, many healthcare providers do not consider surgical intervention as viable option, because of the shortened life expectancy associated with Trisomy 18. Some physicians view surgical intervention as futile and expensive. While the life of the infant may be extended, the physician argues but what is the quality of that life and if the condition carries a not compatible with life diagnosis then why aggressively intervene when the expected outcome with or without an intervention, remains death.

\section{Intervention}

Although Trisomy 18 is a lethal condition and interventions that were once considered futile in this population, thanks to improved medical technology along with more medical options allows for healthcare providers to offer families options instead of just pending death. 
Healthcare providers often struggle with providing aggressive intervention such as surgical repairs when the diagnosis is very poor. In the past when a diagnosis of Trisomy 18 was determined the only possible options healthcare providers offered these families were: termination of pregnancy during the first trimester or continue the pregnancy term and provide comfort care for the infant. Today, the treatment options include previous stated, along with aggressive invasive interventions such as intubation with mechanical ventilation and multiple surgical repairs to correct structural anomalies. The reason for physicians and healthcare professional's willingness to offer aggressive interventions is the improvements in medicine, which the literature has revealed studies that indicate that these children are living longer with the help of aggressive interventions. A retrospective study conducted by McGraw \& Perlman [4], of hospitalized patients ages 0 to 20 with the diagnosis of Trisomy 18 discovered that these children were living longer largely in part to the medical and surgical treatments they were receiving.

\section{Author's Position}

The following sections presented the perspective of the healthcare providers and the perspective of the families in the case of children with terminal illness such as Trisomy 18. It is evident that ethical dilemmas are multifactorial and complex, while there is no right or wrong answers when caring for these children with a diagnosis of not compatible with life. While the author of this paper understands both positions, the challenge lies in choosing one and believing that one is better than the other. Due to the pressing ethical debate with a diagnosis of Trisomy 18 there needs to be ongoing conversations between the families of the child and the healthcare team to ensure that the focus remains with upholding the best interest of the child.

With the improvement in treatment approaches medical science has opened the door for parents and physicians to explore alternative treatment plans that allow the child with a lethal congenital condition a chance at life while allowing the families to experience the opportunity with their child. The literature indicates that healthcare providers and the families of these children recognize and share the same challenge, giving these vulnerable children a chance versus having the infant go through the complexities of multiple surgeries and frequent hospitalizations, all which can drastically impact the child's quality of life. If a family of a Trisomy infant requests interventions then, some factors should be evaluated in order to ensure benefits to the patient is achieved and harm is avoided. It is noted in the literature that the chromosomal anomaly associated with Trisomy 18 varies from full, partial and mosaic.

Therefore, depending on degree of severity of the condition, an intervention may significantly extend not only the life of the infant but the quality as well. Thus, those results would be priceless for the child and their families. Therefore, to conclude healthcare providers defend their decision not to offer interventions based on scientific evidence that asserts that a diagnosis of Trisomy 18 is not compatible with life. Consequently, the families of these children should be allowed the opportunity to have their perspective presented and evaluated at the ethical decision making table. The recommendations of healthcare providers stem from science; however when there are outlier cases of children with Trisomy 18 or any other diagnosis of incompatibility with life, that is the silver lining that gives the families hope. While the expertise of medical professionals is valued and respected, then why not grant the same consideration to the families of these children.

\section{References}

1. Santucci G, Battista V, Kang T (2014) Caring for the infant with Trisomy 18: The bioethical implications of treatment decisions on nurses. J Hosp Palliat Nurs 16: 388-393.

2. Bruns AD (2013) Caring for infant with Trisomy 18: A case study with guidelines. Clin Nurs Stud 4: 30-36.

3. Cereda A, Carey JC (2012) The Trisomy 18 syndrome. Orphanet J of Rare Dis 7: 81 .

4. McGraw MP, Perlman JM (2008) Attitudes of neonatologist toward delivery room management of confirmed Trisomy 18: Potential factors influencing a changing dynamic. Pedia 121: 1106-1110. 